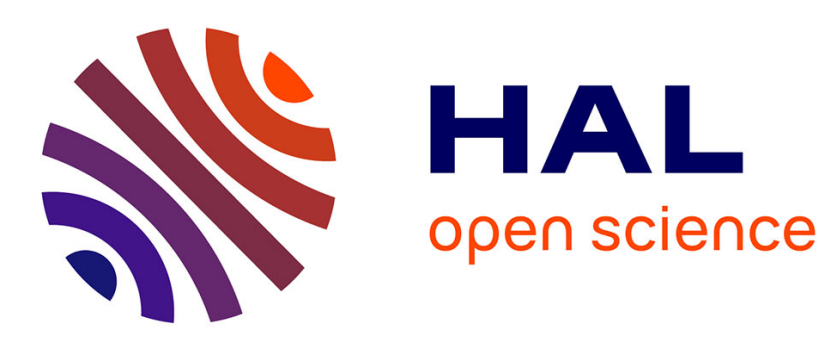

\title{
Vibrations of Poroelastic Plates: Mixed Displacement-Pressure Modelisation and Experiments
}

\author{
Manuel Etchessahar, Sohbi Sahraoui, Bruno Brouard
}

\section{To cite this version:}

Manuel Etchessahar, Sohbi Sahraoui, Bruno Brouard. Vibrations of Poroelastic Plates: Mixed Displacement-Pressure Modelisation and Experiments. Acta Acustica united with Acustica, 2009, 95 (5), pp.857-865. 10.3813/AAA.918217 . hal-00438087

\section{HAL Id: hal-00438087 https://hal.science/hal-00438087}

Submitted on 2 Dec 2009

HAL is a multi-disciplinary open access archive for the deposit and dissemination of scientific research documents, whether they are published or not. The documents may come from teaching and research institutions in France or abroad, or from public or private research centers.
L'archive ouverte pluridisciplinaire HAL, est destinée au dépôt et à la diffusion de documents scientifiques de niveau recherche, publiés ou non, émanant des établissements d'enseignement et de recherche français ou étrangers, des laboratoires publics ou privés. 


\title{
Vibrations of poroelastic plates: mixed displacement-pressure mod- elisation and experiments
}

\author{
Manuel Etchessahar ${ }^{1}$, Sohbi Sahraoui, Bruno Brouard ${ }^{2}$ \\ Laboratoire d'Acoustique de l'Université du Maine-UMR CNRS 6613 \\ Université du Maine, Avenue Olivier Messiaen \\ 72085 Le Mans Cedex 9, FRANCE
}

December 2, 2009

Running title: Vibrations of poroelastic plates

\begin{abstract}
This paper presents the equations of motion of air saturated rectangular poroelastic plates. The model is based on a mixed displacement-pressure formulation of Biot's theory. Two equations of motion are obtained and solved with the Galerkin method for any boundary conditions. These equations take into account the solid-fluid coupling effects. Simulations of the bending vibrations of a rectangular water saturated sandstone and air saturated acoustic foam are performed for studying the influence of the viscous damping through the permeability. Experiments on clamped plates made of low density acoustic materials (fibrous and polyurethane foam) are used to check the limits of this model. On these materials the structural damping is predominant compared to viscous damping.
\end{abstract}

PACS numbers: $43.40 \mathrm{Dx}, 43.20 \mathrm{~Tb}$ [ANN]

\section{Introduction}

In automobile and aircraft applications, plates of porous materials are widely used for passive sound absorption, noise control and vibration reduction. When these plates are bonded onto a vibrating structure or are excited at strong sound levels, the skeleton of the porous material cannot be considered anymore as rigid and its acoustical behaviour can be described by Biot's theory [1]. In the vicinity of resonances of porous plates, relative fluid-structure velocity can lead to high viscous damping [2] and simplified models based on equivalent solid plate [3] or on equivalent fluid description [4] cannot be used anymore.

Some analytical models consisting in solving the general problem of the flexural vibrations of a thin fluid saturated poroelastic plate including Biot's theory are available in the literature. The first models [5][6], motivated by biomedical and geomechanical problems, are based on classical plate theory with Kirchhoff's assumptions and the $[u, U]$ formulation of Biot's relations. More recently, Leclaire et al. [7] used a $[u, u-U]$ formulation of Biot's equations to derive two equations of equilibrium for the porous plate. The main advantages of this model are the simplicity of the equations, and their capability to be solved under boundary conditions other than simply-supported edges. However, these equations were solved with the assumption of a predominant constant transverse fluid-solid relative displacement which influences directly the viscous dissipation within the plate. The model has been validated by experiments on high density clamped porous plates [8].

\footnotetext{
${ }^{1}$ Current address: PSA Peugeot Citroen, 78943 Vélizy-Villacoublay, France

${ }^{2}$ Corresponding author, e-mail: bruno.brouard@univ-lemans.fr
} 
In this paper, a $[u, p]$ formulation of Biot's equations is used in association with the classical theory of plates to derive two coupled equations of equilibrium of an homogeneous and isotropic poroelastic plate [9]. The use of this theory is valid in the case of porous materials as long as the wavelength of the bending waves is much greater than the size of the pores. The fluid loading is not considered and only solid-fluid interactions within the plate are modeled. The plate is supposed to be excited on its solid phase by a punctual force. The equations are solved using the Galerkin method. The transverse solid displacement and the fluid pressure are decomposed on a basis of eigenfunctions chosen as a product of beam functions which verify all the boundary conditions. Hence, various boundary conditions can be investigated: simply supported, clamped or free edges on the solid phase, permeable and impermeable on the fluid phase. The main advantages of this formulation are the simplicity of the two resulting equations, their easy physical interpretation and the fact that the chosen variables are the measurable ones.

In the first part of this paper, $[u, p]$ formulation of the equations of motion of a poroelastic plate is derived and solved with the Galerkin method for any boundary conditions. In the second part, numerical simulations on a water saturated sandstone are produced with this new model and tendencies for variations of the permeability are observed. Some new numerical simulations are made on a polymeric foam, classically used as sound absorbing material, for various values of the permeability. Finally, in the third part, some experiments made on clamped poroelastic plates are compared with numerical simulations resulting from this model. These experiments are performed on light materials currently used in automotive or aircraft sound absorbing applications. This new results are useful to check the limitations of such models.

\section{Theory}

\subsection{The $[u, p]$ formulation of Biot's equations}

The modeling of poroelastic materials is based on Biot's equations of poroelasticity [1], linking the solid and fluid displacements vectors, $\underline{u}$ and $\underline{U}$ respectively, to the stress tensors $\underline{\underline{\sigma}}^{s}$ and $\phi \underline{\nabla} p$ of the solid and fluid phases respectively. Assuming harmonic time dependence is $e^{j \bar{\omega} t}$, equation of motion of a poroelastic medium can be written in the $[u, U]$ form as follows [10]:

$$
\begin{aligned}
& \operatorname{div} \underline{\underline{\sigma}}^{s}+\omega^{2}\left(\tilde{\rho}_{11} \underline{u}+\tilde{\rho}_{12} \underline{U}\right)=0, \\
& -\phi \underline{\nabla} p+\omega^{2}\left(\tilde{\rho}_{12} \underline{u}+\tilde{\rho}_{22} \underline{U}\right)=0,
\end{aligned},
$$

where $\phi$ is the porosity of the porous medium and where the effective densities

$$
\tilde{\rho}_{11}=\rho_{11}+\frac{b(\omega)}{j \omega}, \quad \tilde{\rho}_{22}=\rho_{22}+\frac{b(\omega)}{j \omega}, \quad \tilde{\rho}_{12}=\rho_{12}-\frac{b(\omega)}{j \omega},
$$

are related to the solid and fluid densities, $\rho_{11}$ and $\rho_{22}$ respectively, and to an inertial coupling term $\rho_{12}$. The viscous effects are taken into account in the $b(\omega)$ term which can be written:

$$
b(\omega)=-\phi^{2} \sigma G(\omega),
$$

where $\sigma$ is the flow resistivity and $G(\omega)$ is the Johnson function [11]. A detailed description of all these terms can be found in reference [10].

Using the stress-strain relations defined by Biot [1], equations (1) and (2) can be rewritten in a mixed $[u, p]$ form as follows [12]:

$$
\begin{gathered}
\operatorname{div}{\underline{\underline{\hat{\sigma}^{s}}}}^{s}+\tilde{\rho} \omega^{2} \underline{u}+\tilde{\gamma} \underline{\nabla} p=0, \\
\Delta p+\frac{\tilde{\rho}_{22}}{\tilde{R}} \omega^{2} p-\frac{\tilde{\rho}_{22}}{\phi^{2}} \tilde{\gamma} \omega^{2} d i v \underline{u}=0,
\end{gathered}
$$


where $\tilde{\rho}$ is a reduced effective density

$$
\tilde{\rho}=\tilde{\rho}_{11}-\frac{\tilde{\rho}_{12}^{2}}{\tilde{\rho}_{22}}
$$

and $\tilde{\gamma}$ is a complex number defined by

$$
\tilde{\gamma}=\phi\left(\frac{\tilde{\rho}_{12}}{\tilde{\rho}_{22}}-\frac{\tilde{Q}}{\tilde{R}}\right)
$$

where $\tilde{R}$ and $\tilde{Q}$ are elastic coefficients. The stress tensor $\underline{\underline{\hat{\sigma}}}^{s}$, defined by the relation:

$$
\underline{\underline{\sigma}}^{s}={\underline{\underline{\sigma}}}^{s}-\phi \frac{\tilde{Q}}{\tilde{R}} p \underline{\underline{1}}
$$

is linked to the solid phase strain by the elastic tensor of the skeleton. The elastic constant of the saturating fluid $\tilde{R}$ and the coupling elastic coefficient $\tilde{Q}$ are related to the bulk modulus of the skeleton $K_{b}$, to the bulk modulus of the solid from which the skeleton is made $K_{s}$ and to the bulk modulus of the saturating fluid $K_{f}$ by relations:

$$
\begin{aligned}
\tilde{R} & =\frac{\phi^{2} K_{s}}{1-\phi-K_{b} / K_{s}+\phi K_{s} / K_{f}}, \\
\tilde{Q} & =\frac{\phi K_{s}\left(1-\phi-K_{b} / K_{s}\right)}{1-\phi-K_{b} / K_{s}+\phi K_{s} / K_{f}} .
\end{aligned}
$$

Next, the mixed $[u, p]$ formulation of Biot's equations of poroelasticity, represented by the set of equations (5) and (6), is used to describe the dynamic behaviour of the porous medium.

\subsection{Coupled equations of motion of a poroelastic plate}

\subsubsection{Geometric assumptions and boundary conditions}

Consider a rectangular, isotropic, homogeneous poroelastic plate of lateral dimensions $a \times b$ and of uniform thickness $h$. The system of coordinates is chosen in order to make the $(\vec{x}, \vec{y})$ plane coincide with the middle surface of the poroelastic plate before deformation (Figure 1 ). By following Kirchhoff's assumption [13], the solid displacement vector $\underline{u}=(u, v, w)$ is then a function of the transverse displacement $w(x, y)$, and the in-plane displacements $u$ and $v$ can be written:

$$
u=-z \frac{\partial w(x, y)}{\partial x}, \quad v=-z \frac{\partial w(x, y)}{\partial y} .
$$

By giving that form to the solid displacement, we assume that normal to the middle surface remains straight and normal to the middle surface after deformation. This implies that the saturating fluid inside the pores has no effects on the cross section of the plate.

The poroelastic plate is supposed to be excited on its solid phase by a punctual force $F_{0}$ at coordinates $\left(x_{0}, y_{0}\right)$. In the absence of external acoustic sources and neglecting the sound radiation of the plate, boundary conditions on upper and lower surfaces are:

$$
\begin{aligned}
& z=\frac{h}{2} \quad, \quad \hat{\sigma}_{z z}^{s}=F_{0} \delta\left(x-x_{0}\right) \delta\left(y-y_{0}\right) \quad, \quad \hat{\sigma}_{x z}^{s}=\hat{\sigma}_{y z}^{s}=0 \\
& z=-\frac{h}{2}, \quad \hat{\sigma}_{z z}^{s}=0 \quad, \quad \hat{\sigma}_{x z}^{s}=\hat{\sigma}_{y z}^{s}=0
\end{aligned}
$$

where $\delta$ is the Dirac function. 


\subsubsection{Equilibrium}

The three equations of equilibrium for the solid phase of the poroelastic plate are established following the procedure used by Taber [5]. To do so, the expression of the solid displacement vector $\underline{u}$ of the plate (12) is introduced in the first equation of the mixed $[u, p]$ formulation of Biot's equations of poroelasticity (5). The first two equations of equilibrium are obtained by multiplying by $z$ the modified (5) along $x$ and $y$ axis and by integrating across the plate thickness:

$$
\begin{aligned}
& -\tilde{\rho} \omega^{2} \int_{-\frac{h}{2}}^{\frac{h}{2}} z^{2} \frac{\partial w}{\partial x} d z+\tilde{\gamma} \int_{-\frac{h}{2}}^{\frac{h}{2}} z \frac{\partial p}{\partial x} d z=Q_{x z}-M_{x x, x}-M_{x y, y}, \\
& -\tilde{\rho} \omega^{2} \int_{-\frac{h}{2}}^{\frac{h}{2}} z^{2} \frac{\partial w}{\partial y} d z+\tilde{\gamma} \int_{-\frac{h}{2}}^{\frac{h}{2}} z \frac{\partial p}{\partial y} d z=Q_{y z}-M_{y x, x}-M_{y y, y},
\end{aligned}
$$

where the bending moments $M_{\alpha \beta}$ and the shear resultants $Q_{\alpha z}$ are given by [14]:

$$
M_{\alpha \beta}=\int_{-\frac{h}{2}}^{\frac{h}{2}} \hat{\sigma}_{\alpha \beta}^{s} z d z \quad \text { and } \quad Q_{\alpha z}=\int_{-\frac{h}{2}}^{\frac{h}{2}} \hat{\sigma}_{\alpha z}^{s} d z .
$$

The third equation is obtained by integrating across the plate thickness the modified equation (5) along $z$ axis:

$$
-\tilde{\rho} \omega^{2} \int_{-\frac{h}{2}}^{\frac{h}{2}} w d z+\tilde{\gamma} \int_{-\frac{h}{2}}^{\frac{h}{2}} \frac{\partial p}{\partial z} d z=-Q_{x z, x}-Q_{y z, y}-\left[\hat{\sigma}_{z z}^{s}\right]_{-\frac{h}{2}}^{\frac{h}{2}},
$$

where the latter term is fixed by the boundary conditions on the solid phase of the poroelastic plate (equations (13) and (14)).

\subsubsection{The poroelastic plate equations}

By neglecting the rotary inertia of the plate (first terms of equations (15),(16)), the combination of equations (15), (16), (18) gives the equation of motion of the solid phase of the poroelastic plate:

$$
\tilde{D} \Delta^{2} w(x, y)-\tilde{\rho} h \omega^{2} w(x, y)-\tilde{\gamma} \int_{-\frac{h}{2}}^{\frac{h}{2}}\left(\frac{\partial^{2}}{\partial x^{2}}+\frac{\partial^{2}}{\partial y^{2}}\right) p(x, y, z) z d z=F_{0} \delta\left(x-x_{0}\right) \delta\left(y-y_{0}\right),
$$

where the complex flexural modulus of the skeleton $\tilde{D}$ is related to the complex Young modulus $\tilde{E}$ and to the Poisson's ratio $\nu$ by relation:

$$
\tilde{D}=\frac{\tilde{E} h^{3}}{12\left(1-\nu^{2}\right)} .
$$

The equation for the fluid pressure is obtained by introducing expression (12) of the solid displacement vector $\underline{u}$ in $(6)$

$$
\Delta p(x, y, z)+\frac{\tilde{\rho}_{22}}{\tilde{R}} \omega^{2} p(x, y, z)+\frac{\tilde{\rho}_{22}}{\phi^{2}} \tilde{\gamma} \omega^{2} z \Delta w(x, y)=0 .
$$

The set of equations (19) and (21) are the coupled equations of the poroelastic plate in $[u, p]$ formulation. One of the main advantages of this formulation is the easy understanding of the equations. The first two terms of the solid phase equation (19) are the elastic and inertial terms of the in vacuo classical plate equation while the first two terms of the fluid pressure equation 
(21) represent the fluid propagation inside the poroelastic plate. The third term of each equation is a coupling term which can be considered as a source term.

\subsection{Modal resolution of the coupled equations}

\subsubsection{Galerkin approximation}

The system of coupled equations is solved using the Galerkin method [15]. Dimensionless space variables $\zeta, \eta$ and $\gamma$ are used:

$$
\zeta=\frac{x}{a}, \quad \eta=\frac{y}{b} \quad \text { and } \quad \gamma=\frac{2 z}{h} .
$$

Hence, solutions for the solid transverse displacement $w(x, y)$ and for the fluid pressure $p(x, y, z)$ are approximated respectively by the function $\tilde{w}(\zeta, \eta)$ and $\tilde{p}(\zeta, \eta, \gamma)$ such as:

$$
\tilde{w}(\zeta, \eta)=A_{m n} w_{m n}(\zeta, \eta) \quad \text { and } \quad \tilde{p}(\zeta, \eta, \gamma)=B_{q r s} p_{q r s}(\zeta, \eta, \gamma)
$$

where the test functions $w_{m n}(\zeta, \eta)$ and $p_{q r s}(\zeta, \eta, \gamma)$ fulfill all the boundary conditions and where the coefficients $A_{m n}$ and $B_{q r s}$ are to be determined. Einstein's convention is used for repeated indices $m, n, q, r, s$ varying respectively from 1 to $m_{0}, n_{0}, q_{0}, r_{0}, s_{0}$. Applying Galerkin method [15] on equations (19) and (21) yields to the following linear system of equations:

$$
\begin{gathered}
\iint\left(L_{11} \tilde{w}+L_{12} \tilde{p}\right) w_{i j} d \zeta d \eta=\iint F_{0} \delta\left(\zeta_{0}, \eta_{0}\right) w_{i j} d \zeta d \eta \quad \text { for } \quad(i, j) \in\left(1 \cdots m_{0}\right) \times\left(1 \cdots n_{0}\right) \\
\iiint\left(L_{21} \tilde{w}+L_{22} \tilde{p}\right) p_{i j k} d \zeta d \eta d \gamma=0 \quad \text { for } \quad(i, j, k) \in\left(1 \cdots m_{0}\right) \times\left(1 \cdots n_{0}\right) \times\left(1 \cdots s_{0}\right)
\end{gathered}
$$

where the $L_{\alpha \beta}$ are the corresponding linear operators of equations (19) and (21). The resolution of this system leads to the unknown $A_{m n}$ and $B_{q r s}$ coefficients.

\subsubsection{Choice of the set of test functions}

The transverse solid displacement test functions $w_{m n}(\zeta, \eta)$ were chosen as :

$$
w_{m n}(\zeta, \eta)=\phi_{m}(\zeta) \psi_{n}(\eta) .
$$

where $\phi_{m}(\zeta)$ and $\psi_{n}(\eta)$ are the so-called beam eigenfunctions verifying all the boundary conditions along $\zeta$ and $\eta$ axis respectively [14]. The literature on the mathematical form of these beam eigenfunctions is abundant and combinations of trigonometric and hyperbolic functions [16] or combinations of trigonometric and polynomial functions [17] can be found. More recently, sets of orthogonal polynomial functions [18] or hierarchical sets based on polynomial [19] or trigonometric [20] functions were introduced in order to improve the efficiency of numerical methods. In this paper a classical set based on trigonometric and hyperbolic functions is used for $\phi_{m}(\zeta)$ and $\psi_{n}(\eta)$.

In addition to the solid displacement, the fluid pressure test functions $p_{q r s}(\zeta, \eta, \gamma)$ are written as:

$$
p_{q r s}(\zeta, \eta, \gamma)=\phi_{q}(\zeta) \psi_{r}(\eta) \theta_{s}(\gamma)
$$

where $\phi_{q}(\zeta)$ and $\psi_{r}(\eta)$ are the beam functions chosen for the solid displacement, and the function $\theta_{s}(\gamma)$ is related to the transverse pressure slope. We suppose here that the pressure follows the form of $w_{m n}(\zeta, \eta)$. Two types of boundary conditions on the upper and lower surfaces, leading to two sets of transverse slope eigenfunctions, are explored: permeable and impermeable surfaces. 
Note that these two types of boundary conditions describe perfectly poroelastic materials used usually in sound absorbing applications: ordinary foams and fibrous materials can be considered pervious while acoustic materials with surface treatment (baked or finished) can be considered impervious. For a permeable plate, the pressure must vanish on the upper and lower surfaces and on the neutral fiber, leading to the following form for $\theta_{s}(\gamma)$ :

$$
\theta_{s}(\gamma)=\sin \left(k_{s z} \gamma\right) \quad \text { with } \quad k_{s z}=s \pi \quad \text { and } \quad s=1,2,3, \ldots,
$$

while for an impermeable plate, $\theta_{s}(\gamma)$ takes the form:

$$
\theta_{s}(\gamma)=\sin \left(\frac{k_{s z}}{2} \gamma\right) \quad \text { with } \quad s=1,3,5, \ldots
$$

The fluid pressure slope forms for both pervious and impervious conditions are like those computed by Taber [5].

\subsubsection{Solution of the coupled system}

By substituting displacement $\tilde{w}(\zeta, \eta)$ and pressure $\tilde{p}(\zeta, \eta, \gamma)$ by their expressions (23) in equations (24) and (25), and by taking into account (26) and (27), one can obtain the following linear system of equations for the unknown $A_{m n}$ and $B_{q r s}$ coefficients:

$$
\begin{gathered}
\left\{K_{m n i j}-\omega^{2} M_{m n i j}\right\} A_{m n}-C_{q r s i j} B_{q r s}=F_{i j} \quad \text { for } \quad(i, j) \in\left(1 \cdots m_{0}\right) \times\left(1 \cdots n_{0}\right), \\
\left\{L_{q r s i j k}+\omega^{2} N_{q r s i j k}\right\} B_{q r s}+\omega^{2} D_{m n i j k} A_{m n}=0 \quad \text { for } \quad(i, j, k) \in\left(1 \cdots m_{0}\right) \times\left(1 \cdots n_{0}\right) \times\left(1 \cdots s_{0}\right),
\end{gathered}
$$

where $K_{m n i j}$ and $L_{q r s i j k}$ are the stiffness coefficients of the solid and fluid phases, $M_{m n i j}$ and $N_{q r s i j k}$ are the mass coefficients, the $C_{q r s i j k}$ and $D_{m n i j}$ are related to the damping and mass coupling between the two phases, and $F_{i j}$ are the imposed force coefficients. These coefficients are defined by:

$$
\begin{gathered}
K_{m n i j}=\tilde{D} a b\left[\frac{1}{a^{4}} I_{m i}^{40} I_{n j}^{00}+\frac{1}{b^{4}} I_{m i}^{00} I_{n j}^{40}+\frac{2}{a^{2} b^{2}} I_{m i}^{20} I_{n j}^{20}\right] \\
L_{q r s i j k}=\tilde{R} a b h\left[\frac{1}{a^{2}} I_{q i}^{20} I_{r j}^{00} I_{s k}^{00}+\frac{1}{b^{2}} I_{q i}^{00} I_{r j}^{20} I_{s k}^{00}+\frac{1}{h^{2}} I_{q i}^{00} I_{r j}^{00} I_{s k}^{20}\right] \\
M_{m n i j}=\tilde{\rho} a b h I_{m i}^{00} I_{n j}^{00} \\
N_{q r s i j k}=\tilde{\rho}_{22} a b h I_{q i}^{00} I_{r j}^{00} I_{s k}^{00} \\
C_{q r s i j}=\tilde{\gamma} a b h t_{s}\left[\frac{1}{a^{2}} I_{q i}^{20} I_{r j}^{00}+\frac{1}{b^{2}} I_{q i}^{00} I_{r j}^{20}\right] \\
D_{m n i j k}=\tilde{R} \frac{\tilde{\rho}_{22}}{\phi^{2}} \tilde{\gamma} a b h^{2} t_{k}\left[\frac{1}{a^{2}} I_{m i}^{20} I_{n j}^{00}+\frac{1}{b^{2}} I_{m i}^{00} I_{n j}^{20}\right] \\
F_{i j}=F_{0} \phi_{i}\left(\zeta_{0}\right) \psi_{j}\left(\eta_{0}\right)
\end{gathered}
$$

where the $I_{s t}^{\alpha \beta}$ and the $t_{\alpha}$ are defined by:

$$
I_{s t}^{\alpha \beta}=\int_{0}^{1} \frac{\partial^{\alpha}}{\partial \zeta^{\alpha}} \phi_{s}(\zeta) \frac{\partial^{\beta}}{\partial \zeta^{\beta}} \phi_{t}(\zeta) d \zeta \quad \text { and } \quad t_{\alpha}=\int_{-1}^{1} \theta_{\alpha}(\gamma) \gamma d \gamma
$$

The system composed by equations (30) and (31) can be written as a matricial system and then easily be solved numerically. 


\section{$3 \quad$ Numerical examples}

This section presents various numerical simulations of simply supported poroelastic plates. As observed in previous works [6] [7], the permeability has a great influence on the first bending mode of a water saturated sandstone. Then, attention is focused on the dynamic behaviour of sound absorbing materials. In fact, unlike the water saturated sandstone, these air-saturated materials are highly porous, soft and highly damped such as inertial, elastic and viscous effects are weaker. Some numerical simulations are made on a representative foam usually used in sound absorbing applications. The influence of the permeability is specially studied under the same boundary conditions (simply supported edges). Results obtained for pervious and impervious plates are compared.

\subsection{Case of a water-saturated sandstone}

A simply supported water-saturated sandstone plate (see properties in Table I), of dimensions $4 \times 4 \times 0.2 \mathrm{~m}$ is excited by a unit point force at coordinates $(0.1 \mathrm{~m}, 0.15 \mathrm{~m})$. In order to increase the influence of viscous effects, the structural damping of the material is supposed to be zero.

Figure 2 presents the normalized displacement $w / w_{\text {static }}$ of the first bending mode at the center of the plate for different values of permeability.

The influence of the permeability on the resonance is due to complex coupling effects between skeleton and saturating fluid. In fact, two limit cases are reached for high and low permeabilities. When the permeability is very high, the fluid is totally free and can go through the pores without any viscous dissipation. When the permeability is very low, the saturating fluid is totally hold so that skeleton and fluid motions are in phase and there is no viscous dissipation. Besides, when the fluid is totally hold, the effective mass and the effective stiffness of the material are changed to $\rho+\delta \rho$ and $K+\delta K$ respectively depending on the properties of the saturating fluid (mass, compressibility and viscosity). As a consequence, the frequency at resonance is shifted either to the right, if the stiffness variation is greater than the mass variation, or to the left on the opposite situation. These variations result from complex fluid-structure coupling effects. Obviously, in the case of a water saturated sandstone this change of behavior is mass-like and the resonant frequency is shifted to lower frequencies. Between these two limits the magnitude reaches through a minimum which is the best compromise of a low permeability and a high fluid-solid relative speed. In the next section, simulations on air-saturated foams will show a small influence of the viscous dissipation in comparison with the structural damping.

\subsection{Case of an air-saturated polymeric foam}

Concerning porous materials usually used in sound absorbing applications, the porosity is fairly higher than 0.9 and the saturating fluid is air so that inertial, elastic and viscous coupling effects are weaker than in the case of a water saturated sandstone. Furthermore, polymeric foams are highly damped materials with a skeleton structural loss factor generally near 0.1 [21] so that structural losses can become the predominant source of dissipation. However, a study on the dissipation mechanisms in a porous layer bonded onto a plate [3] showed that the viscous dissipation within the porous material can be important (up to $80 \%$ of the total dissipation) for soft materials. In addition, Dauchez et al. [3] confirm that these viscous effects were mainly related to permeability in the low frequency range. On these basis, some numerical simulations of simply supported pervious and impervious poroelastic plates of dimensions $0.5 \times 0.5 \times 0.02 \mathrm{~m}$ are presented. The material is an air-saturated polymeric foam, very similar to the one of reference [3], used for sound absorbing applications. Its properties are summarized in Table II. In 
order to quantify the effects of viscous dissipation, the permeability is varied from $10^{-8}$ to $10^{-12}$.

Figure 3 presents the normalized solid displacement at the center of the plate of the first bending mode for various permeabilities. The frequency at resonance is the same for the two boundary conditions. For a pervious plate, viscous dissipation is found to be negligible compared to the structural dissipation for all the tested permeabilities. In the case of an impervious plate, the quality factor decreases significantly for very low values of permeability, and remains unchanged for commons permeabilities. On the basis of these observations, one can conclude that the structural damping is the predominant dissipative phenomenon taking place in the first bending mode of polymeric foam plate.

Figure 4 presents the normalized solid displacement at the center of the plate of a higher mode (fifth for example) for various permeabilities. This figure shows two tendencies. Firstly, the viscous dissipation is found to be more important leading to lower quality factors. The viscous effects are predominant on the impermeable plate like it happened in the first mode. In fact, impermeable surfaces do not let the fluid go out of the plate which increases the fluid structure coupling effects. Secondly, a shift of frequency appears for the impermeable plate. Unlike water saturated sandstone, this shift drifts to higher frequencies. A physical explanation of this effect is that, when the air is hold in the skeleton of the foam, the variation of density (density of air present in the material: $1.16 \mathrm{~kg} / \mathrm{m}^{3}$, density of the skeleton: $39 \mathrm{~kg} / \mathrm{m}^{3}$ ) is very low compared to the variation of stiffness, the bulk modulus of the air being of the same order than the Young's modulus of the skeleton (bulk modulus of air: $1.410^{5} \mathrm{~Pa}$, Young's modulus of the skeleton: $2.0610^{5} \mathrm{~Pa}$ ).

\section{Experiments}

In this section some experiments are performed on two air saturated poroelastic plates made of materials used for passive sound absorption purposes. The first plate is made of fibers while the second one is made of polymeric foam. Since these materials present higher porosities and weaker Young's moduli (see properties in Table II) than the materials used by Leclaire et al. [8], the effects of the saturating fluid will be more important. The experimental set-up used for the measurements of the frequency response of the plate is described first. In order to compute a good frequency response function (FRF) classically used in experimental modal testing, both input and output signals must be correctly measured. The measurement difficulties relevant to the nature of such highly porous and weakly stiffened materials are thus described, attention being focused on the measurement of the input force. Finally, experimental results are compared to numerical ones computed with the present model.

\subsection{Experimental set-up}

The experimental set-up is shown in Figure 5. The porous sample of lateral dimensions $0.25 \times 0.22$ $\mathrm{m}$ is clamped at its four edges by the way of a steel jaw smoothly gripped on a small part of the surfaces. The steel frame supporting the porous plate is supposed to be heavy enough to be motionless during experiments. The sample is excited by a punctual force produced by an electrodynamic shaker, the input force $F$ and the acceleration $A$ signals being measured by the way of an impedance head. The input signal is a sweep sine generated by an FFT analyser. The transverse solid phase velocity $V$ of the plate is measured by a laser vibrometer fixed onto a 2 dimensional robot allowing a complete scan of the plate surface. By using a non-contact technique to measure the response of such light and flexible structures we ensure that the response of the plate is not disturbed by an additional punctual mass that could be 
caused by an accelerometer. Both the supporting frame and the shaker are freely supported as advocated in reference [22].

\subsection{Measurements details}

The main difficulty of testing such materials results from the measurement of the input force. Figure 6 shows the driving accelerance $A / F_{c}$ at point $x_{0}=0.174 \mathrm{~m}$ and $y_{0}=0.025 \mathrm{~m}$ of the $0.25 \times 0.22 \times 0.009 \mathrm{~m}$ fibrous clamped plate. The force $F_{c}$ is the force actually applied to the structure calculated according to the process of "mass cancellation" [22]. This figure shows a predominant weakly damped resonance near $200 \mathrm{~Hz}$ and several secondary resonances highly damped from each side of it. Keeping in mind these important differences in damping and noting that the general appearance of the curve is like the one of a Single Degree of Freedom accelerance, it is possible to think that the resonance near $200 \mathrm{~Hz}$ is not due to a bending mode of the plate but to a mass-spring system-like mode. Since the stiffness of the plate is like the stiffness of the excitator, the system excitator+plate forms a SDOF system having its own natural frequency. This analysis has been confirmed by the following experimental observations: the resonance moves to lower frequencies when the driving point is near the center of the plate (lower apparent stiffness) and to upper frequencies when the driving point is near the edges (higher apparent stiffness). Whatever the chosen point is, the mass-spring like resonance remains located near the first bending modes.

\subsection{Comparison between experimental and numerical results}

Measurements were performed on the two clamped plates of dimensions $0.25 \times 0.22 \times 0.009 \mathrm{~m}$. The solid transverse velocities $\dot{w}_{i}$ were measured on 9 points established at the same distance from one another on the plate $(x= \pm 75 \mathrm{~mm}$ and $y= \pm 66 \mathrm{~mm}$ from its center). The reference signal $\dot{w}_{r}$ is the velocity measured at driving point and the chosen indicator is the mean quadratic velocity of the plate defined by:

$$
V=\frac{1}{n} \sum_{i=1}^{n=9}\left|\dot{w}_{i}\right|^{2} /\left|\dot{w}_{r}\right|^{2} .
$$

The acoustical and mechanical properties of the fibrous material and of the polymeric foam used for these experiments are summarized in Table II. The structural loss factors were set to 0.05 and 0.11 respectively according to results found in the literature [23][24]. The first resonant frequency of numerical simulations matches exactly the experimental one as a result of the way both Young's moduli were set.

Figure 7 presents the numerical and experimental mean quadratic velocity of the air saturated fibrous plate excited at point $x_{0}=0.174 \mathrm{~m}$ and $y_{0}=0.025 \mathrm{~m}$. Despite an over estimation of magnitude of the first mode, a good agreement is found between experimental and numerical results specially up to the third mode. For upper modes, a shift of experimental results to lower frequencies and magnitudes at resonance is observed.

Figure 8 presents the numerical and experimental mean quadratic velocity of the air saturated polymeric foam plate excited at point $x_{0}=0.178 \mathrm{~m}$ and $y_{0}=0.078 \mathrm{~m}$. One can easily observe that the experimental curve is much more dumped that the predicted one. In addition, the resonance frequencies are incorrectly predicted. As a consequence, it seems that the developed model does not take into account all the occuring physical phenomenas.

Works on the interactions between structures and their fluid loading [25] have shown that two cases can be distinguished: structures loaded by light fluid and structures loaded by heavy 
fluid. The light fluid assumption, actually made in this modelisation, is classically used when the density of the structure is much more greater than the density of the saturating fluid. In this case the presence of the fluid has no consequences on the structure vibrations and is generally neglected. Otherwise, when the density of the structure is of the same order than the density of the loading fluid (assumption of heavy fluid), the presence of the fluid cannot be neglected anymore. When taking into account the radiation impedance of the structure, added mass and added loss terms appear in the plate equation. This leads to a shift to lower frequencies and magnitudes at resonances. The observations made on the bending vibrations of the fibrous plate, for which the density ratio is near 140, can be explained by this process. Obviously, for very light materials such as polymeric foams the density ratio is near 25 and the assumption of light fluid cannot be made anymore. The loading fluid has a great influence on the vibration of the plate, particularly on the damping. In fact, an analysis of all the phenomena taking place in this case is even more complicated since the elastic constants of such materials are frequency dependent.

\section{Conclusions}

In this paper, we derive a new set of equations for the bending vibrations of poroelastic plates based on the $[u, p]$ formulation of Biot's equations. The first equation governs the bending vibrations of the skeleton while the second one describes the fluid propagation through the plate. These equations have been solved using the Galerkin method and any boundary conditions on the solid phase can be simulated. Solutions for permeable and impermeable plates have also been written. The $[u, p]$ model has been qualitatively validated by reproducing some numerical simulations made by previous authors on a water-saturated sandstone. In addition, a numerical study on light porous materials, such as those usually used in sound absorbing applications, shows that the structural damping is predominant compared to viscous dissipation for the first bending modes. Finally, an experimental validation of the model on these light materials was made. The present model seems well adapted to predict the first resonances of high density porous materials. Nevertheless, the radiation impedance of the material must be integrated to the model for accurate predictions of soft porous materials like polymeric foams. 


\section{References}

[1] M.A. Biot. The theory of propagation of elastic waves in a fluid-satured porous solid. i. low frequency range. ii. higher frequency range. Journal of the Acoustical Society of America, 28:168-191, 1956.

[2] M.J. Swift, K.V. Horoshenkov, P. Leclaire, D.C. Hothersall, K. Fujiwara, and H. Torihama. On the effect of the bending vibration on the acoustic properties of thin poroelastic plates. Journal of the Acoustical Society of America, 107:1786-1789, 2000.

[3] N. Dauchez, S. Sahraoui, and N. Attala. Investigation and modeling of damping in a plate with a bonded porous layer. Journal of Sound and Vibration, 265:437-449, 2003.

[4] K.V. Horoshenkov and K. Sakagami. A method to calculate the acoustic response of a thin, baffled, simply supported poroelastic plate. Journal of the Acoustical Society of America, 110:904-917, 2001.

[5] L.A. Taber. A theory for transverse deflection of poroelastic plates. ASME Journal of Applied Mechanics, 59:628-634, 1992.

[6] D.D. Theodorakopoulos and D.E. Beskos. Flexural vibrations of poroelastic plates. Acta Mechanica, 103:191-203, 1994.

[7] P. Leclaire, K.V. Horoshenkov, and A. Cummings. Transverse vibrations of a thin rectangular porous plate saturated by a fluid. Journal of Sound and Vibrations, 247:1-18, 2001.

[8] P. Leclaire, K.V. Horoshenkov, M.J. Swift, and D.C. Hothersall. The vibrational response of a clamped rectangular porous plate. Journal of Sound and Vibrations, 247:19-31, 2001.

[9] M. Etchessahar, S. Sahraoui, and B.Brouard. Bending vibrations of a rectangular poroelastic plate. C. R. Acad. Sci. Paris, Serie II b, 329:615-620, 2001.

[10] J.F. Allard. Propagation of sound in porous media: modeling sound absorbing materials. Chapman et Hall, Londres, 1993.

[11] D.L. Johnson, J. Koplik, and R. Dashen. Theorie of dynamic permeability and tortuosity in fluid-satured porous media. J. Fluid Mechanics, 98:635-643, 1987.

[12] N. Atalla, R. Panneton, and P. Debergue. A mixed displacement-pressure formulation for poroelastic materials. Journal of the Acoustical Society of America, 104:1444-1452, 1998.

[13] S. P. Timoshenko and S. Woinowsky-Krieger. Theory of plates and shells. McGraw-Hill, New York, 2nd edition, 1959.

[14] A.W. Leissa. Vibration of plates. Acoustical Society of America, Woodbury, NY, 1993.

[15] K. Washisu. Variational Methods in Elasticity and Plasticity. Pergamon Press, Oxford, 1982 .

[16] G.B. Warburton. The vibration of rectangular plates. Proceedings of the Institution of Mechanical Engineers, 168:371-384, 1954.

[17] Ding Zhou. Natural frequencies of rectangular plate using a set of static beam functions in rayleigh-ritz method. Journal of Sound and Vibrations, 189:81-87, 1996. 
[18] R.B. Bhat, S.Chakraverty, and I. Stiharu. Recurrence scheme for the generation of twodimensional boundary characteristic orthogonal polynomials to study vibration of plates. Journal of Sound and Vibrations, 216:321-327, 1998.

[19] N.S. Bardel. Free vibration analysis of a flat plate using the hierarchical finite element method. Journal of Sound and Vibrations, 151:263-289, 1991.

[20] O. Beslin and J. Nicolas. A hierarchical functions set for predicting very high order plate bending modes with any boundary conditions. Journal of Sound and Vibrations, 202:633$655,1997$.

[21] M. Melon, E. Mariez, C. Ayrault, and S. Sahraoui. Acoustical and mechanical characterization of anisotropic open-cell foams. Journal of the Acoustical Society of America, 104:2622-2627, 1998.

[22] D.J. Ewins. Modal testing: theory, practise and application. Research Studies Press, Baldock, England, 2000.

[23] T. Pritz. Dynamic young's modulus and loss factor of plastic foams for impact sound isolation. Journal of Sound Vibrations, 178:315-322, 1994.

[24] M. Mariez and S. Sahraoui. Mesurement of mechanical anisotropic properties of acoustic foams. In Internoise 97, Budapest, Hongrie, 1997.

[25] M.C. Junger and D. Feit. Sound, structures and their interaction. Acoustical Society of America Publications, 1993. 
Table I: Properties of sandstone

\begin{tabular}{|c|c|c||c|c|c|c|}
\hline $\begin{array}{c}\text { porosity } \\
\phi\end{array}$ & Permeability & tortuosity & Young's mod. & struc. loss fact. & Poisson's ratio & solid density \\
$\left.\rho^{2}\right)$ & $\alpha_{\infty}$ & $E(\mathrm{~Pa})$ & $\eta$ & $\nu$ & $\rho_{1}\left(\mathrm{~kg} . \mathrm{m}^{-3}\right)$ \\
\hline \hline 0.3 & {$\left[10^{-4}, 10^{-10}\right]$} & 1.02 & $7.310^{9}$ & 0 & 0.3 & 2300 \\
\hline
\end{tabular}


Table II: Acoustical and mechanical properties of fibrous $\mathrm{F}$ and of polyurethane foam $\mathrm{P}$

\begin{tabular}{|c|c|c|c||c|c|c|c|}
\hline & porosity & Permeability & tortuosity & Young's mod. & struc. loss fact. & Poisson's ratio & solid density \\
& $\phi$ & $K\left(\mathrm{~m}^{2}\right)$ & $\alpha_{\infty}$ & $E(\mathrm{~Pa})$ & $\eta$ & $\nu$ & $\rho_{1}\left({\left.\mathrm{~kg} . \mathrm{m}^{-3}\right)}^{-3}\right.$ \\
\hline \hline $\mathrm{F}$ & 0.95 & $2.1310^{-10}$ & 1.12 & $29.910^{6}$ & 0.05 & 0.3 & 3304 \\
\hline $\mathrm{P}$ & 0.97 & $1.5310^{-10}$ & 1.6 & $16910^{3}$ & 0.11 & 0.3 & 998 \\
\hline
\end{tabular}


FIG. 1 - System of coordinates of the plate.

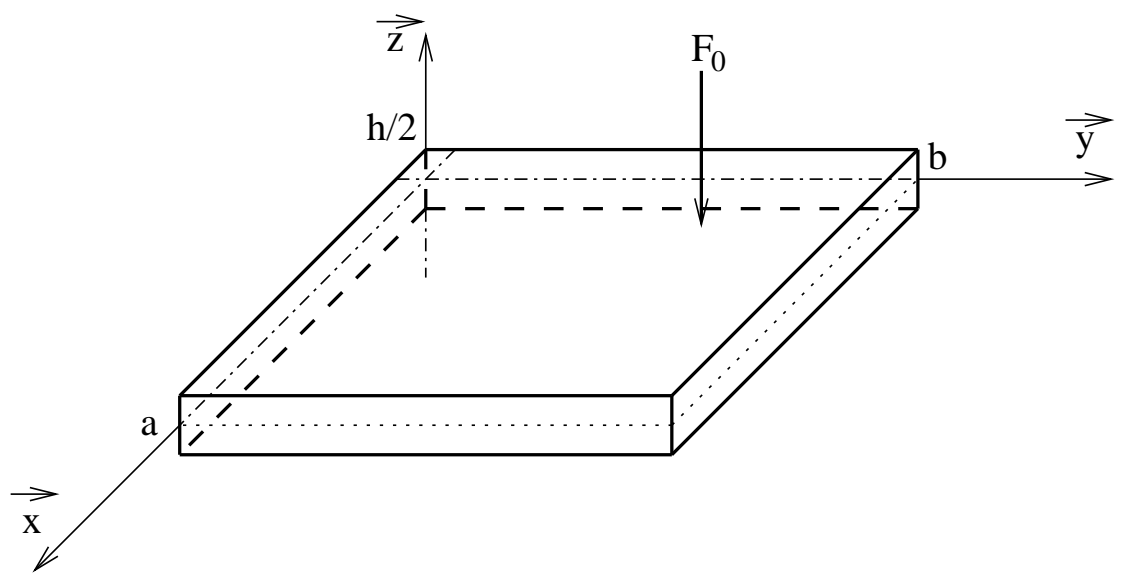

Figure 1: 
FIG. 2 - $|w| /\left|w_{\text {static }}\right|$ of the first resonance of a simply supported water saturated sandstone plate for different values of permeability.

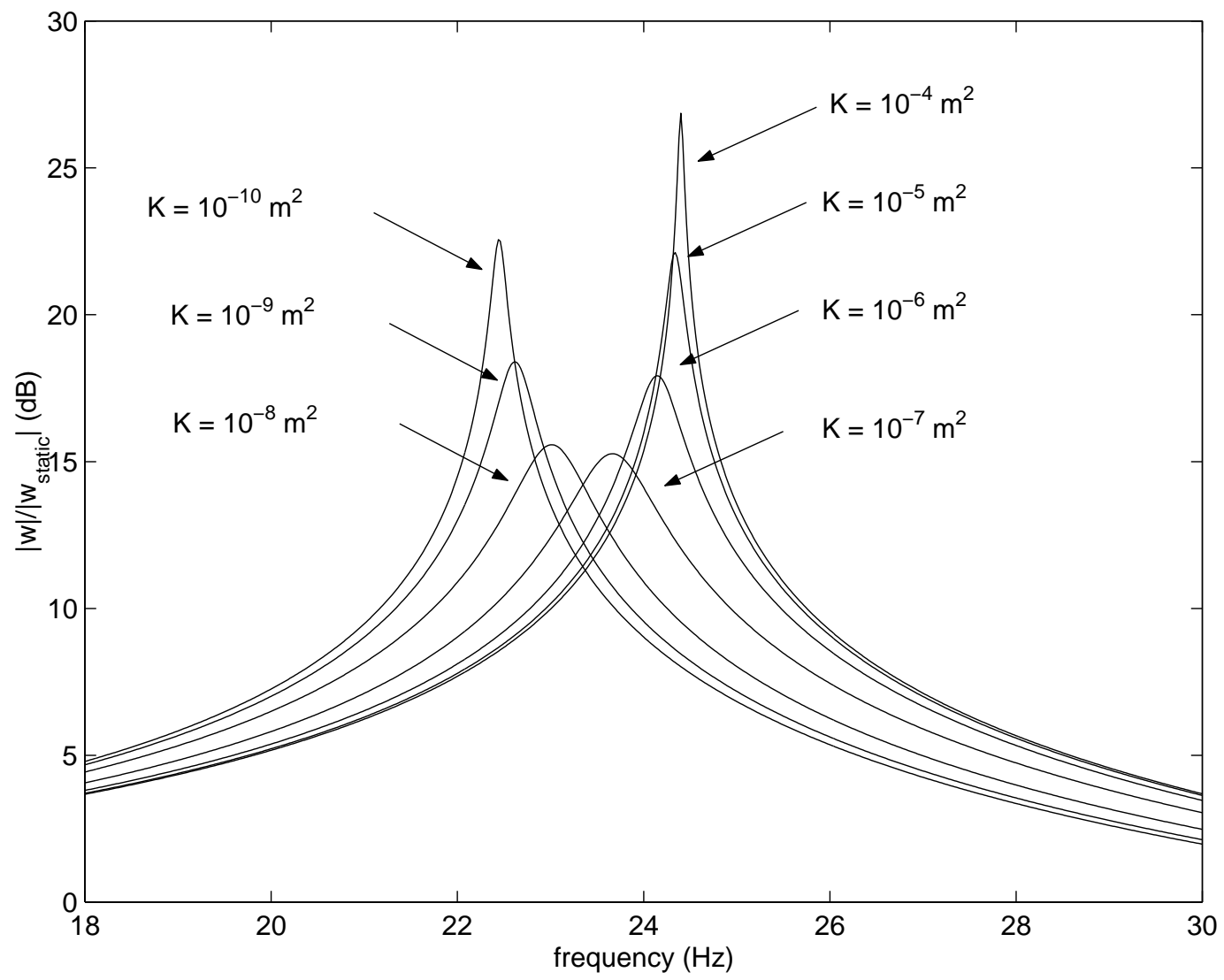

Figure 2: 
Fig. 3 - First bending mode of pervious and impervious plates (figures left and right respectively) for different values of permeability. The numbers $i$ refers to the permeability such as $K=10^{-i} \mathrm{~m}^{2}$.
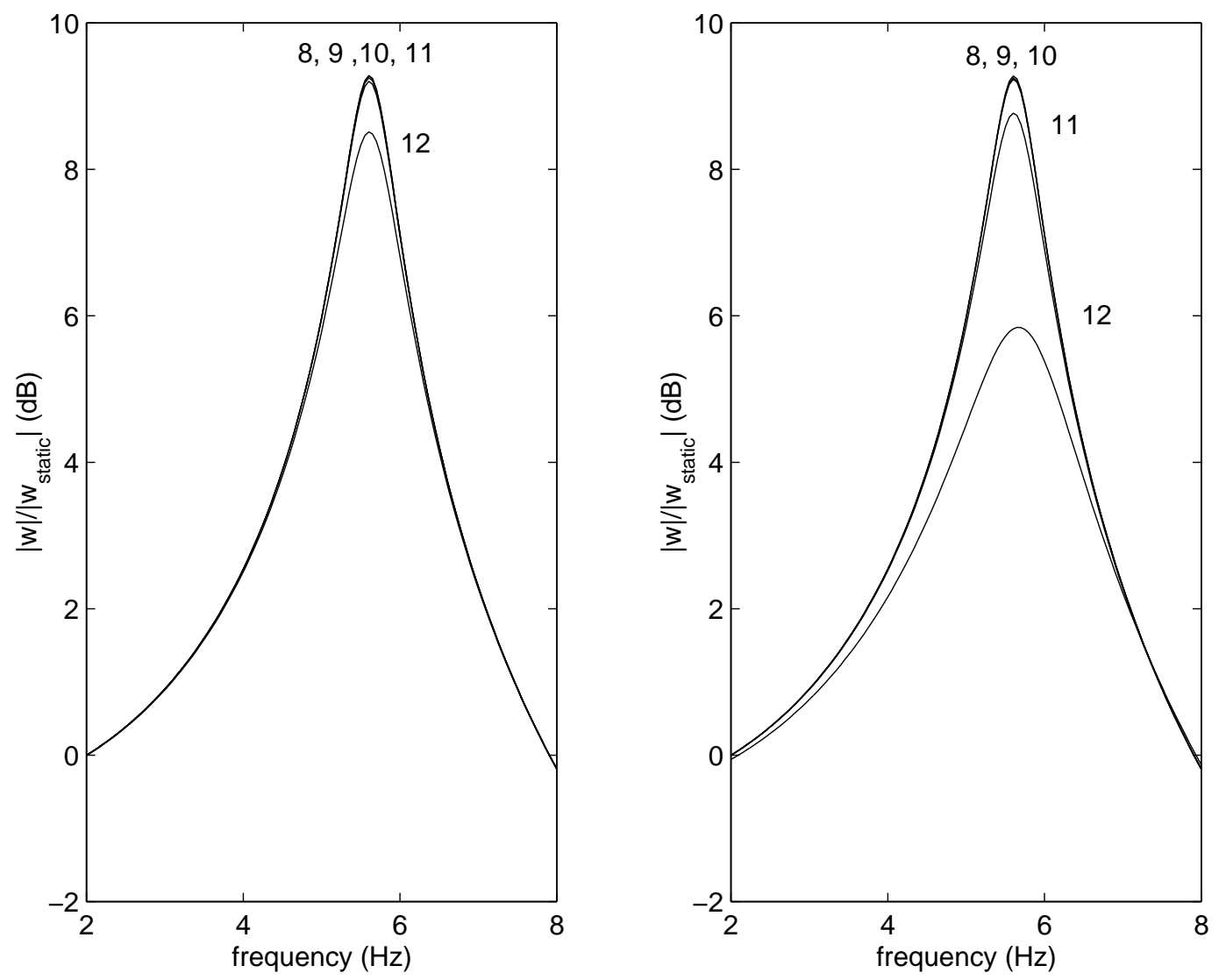

Figure 3: 
Fig. 4 - Fifth bending mode of pervious and impervious plates (figures left and right respectively) for different values of permeability. The numbers $i$ refers to the permeability such as $K=10^{-i} \mathrm{~m}^{2}$.
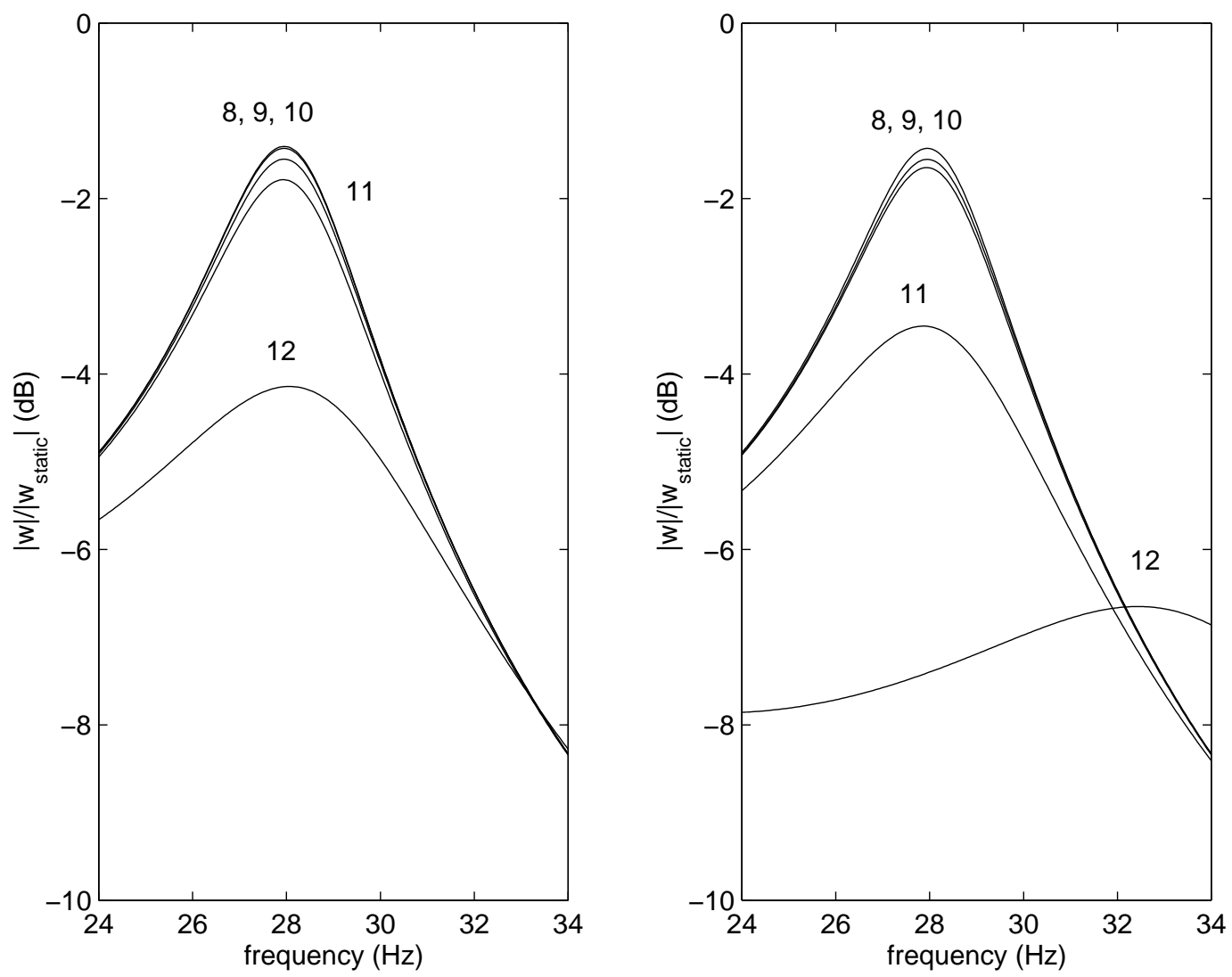

Figure 4: 
FIG. 5 - Experimental set-up.

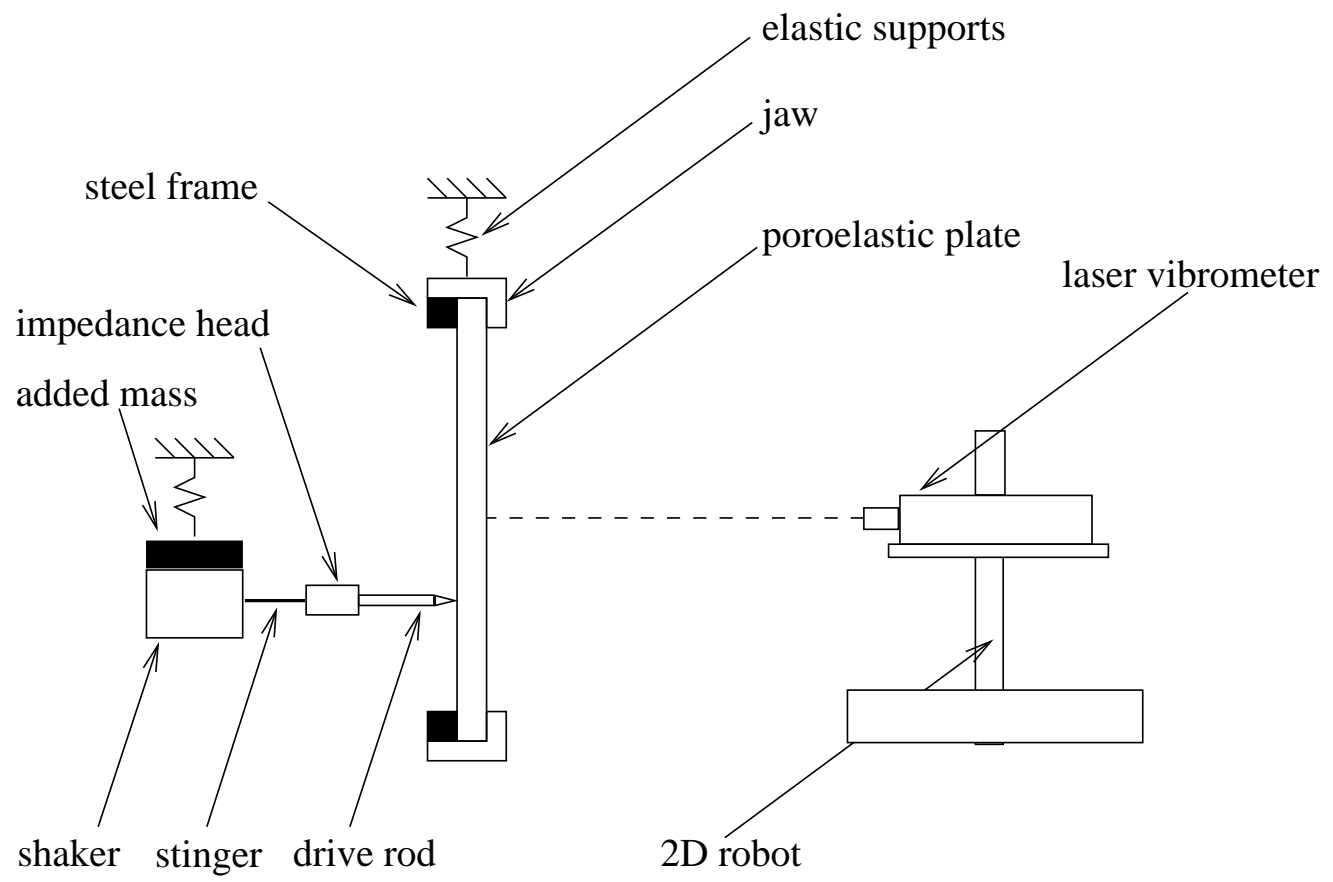

Figure 5: 
Fig. 6 - Transfert function (complex ratio acceleration to applied force) at driving point $x_{0}=0.174 \mathrm{~m}$ and $y_{0}=0.025 \mathrm{~m}$ of a clamped fibrous plate.

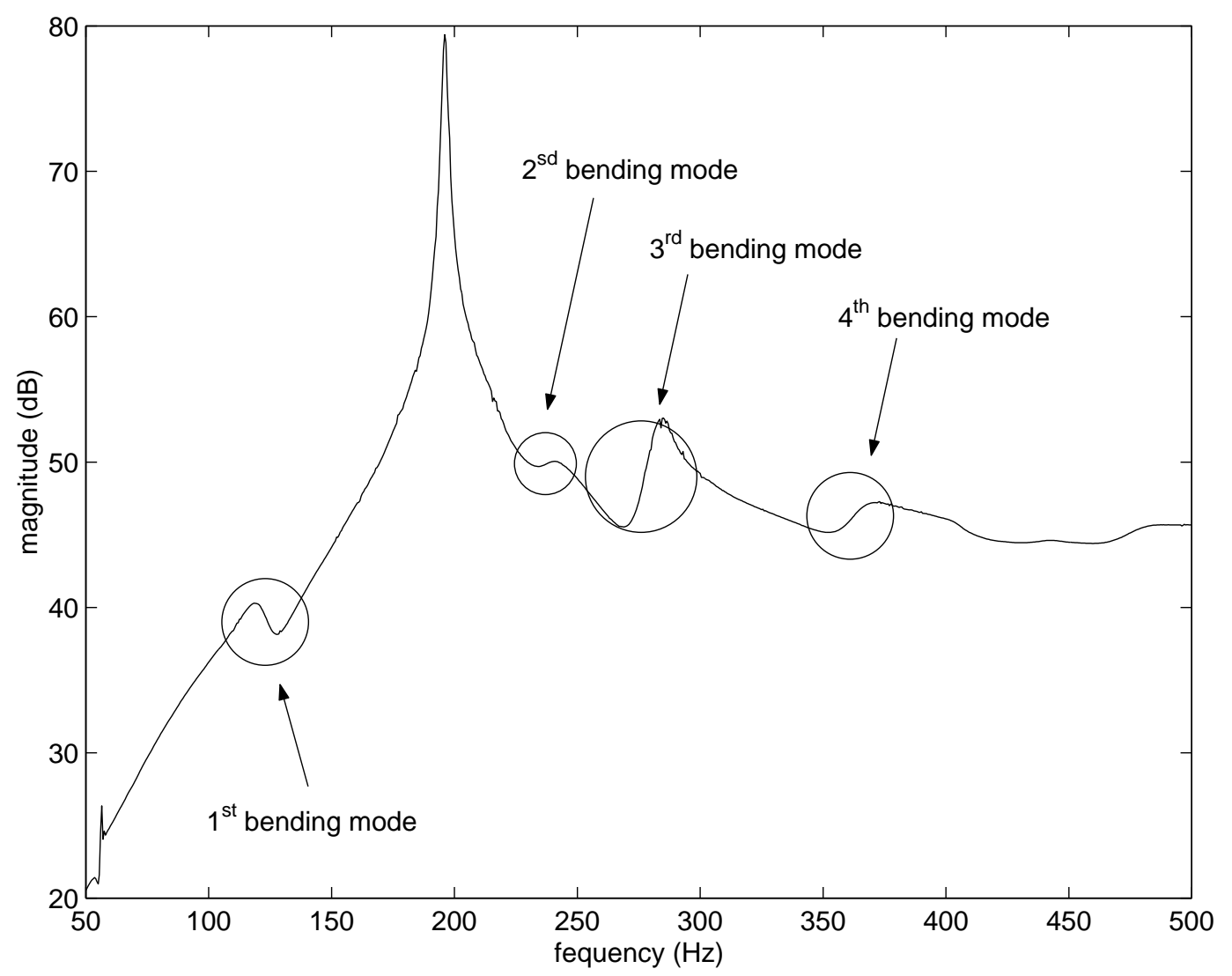

Figure 6: 
FIG. 7 - Mean quadratic velocity of fibrous plate. Comparison between experience and model.

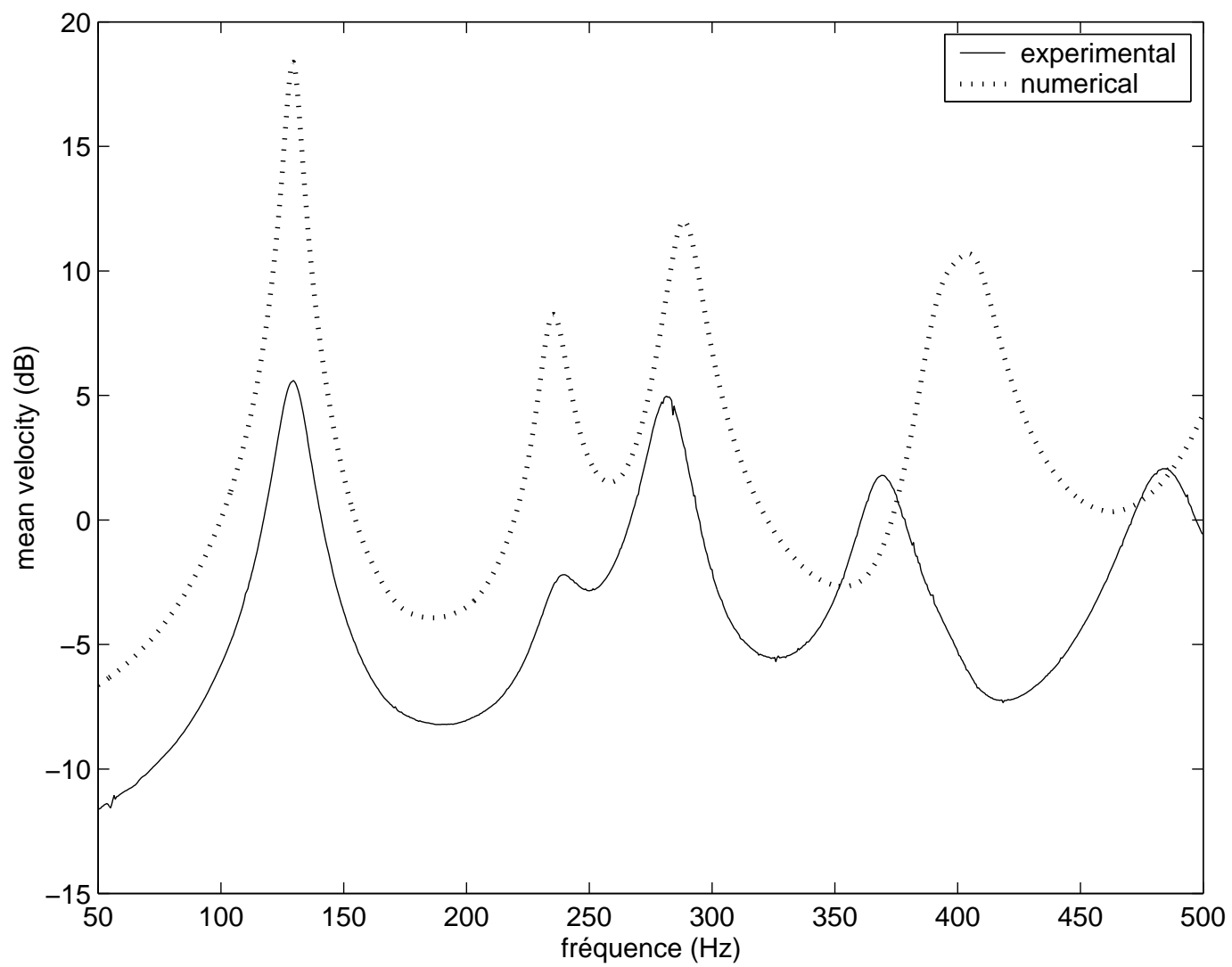

Figure 7: 
Fig. 8 - Mean quadratic velocity of a polyurethane foam plate. Comparison between experience and model.

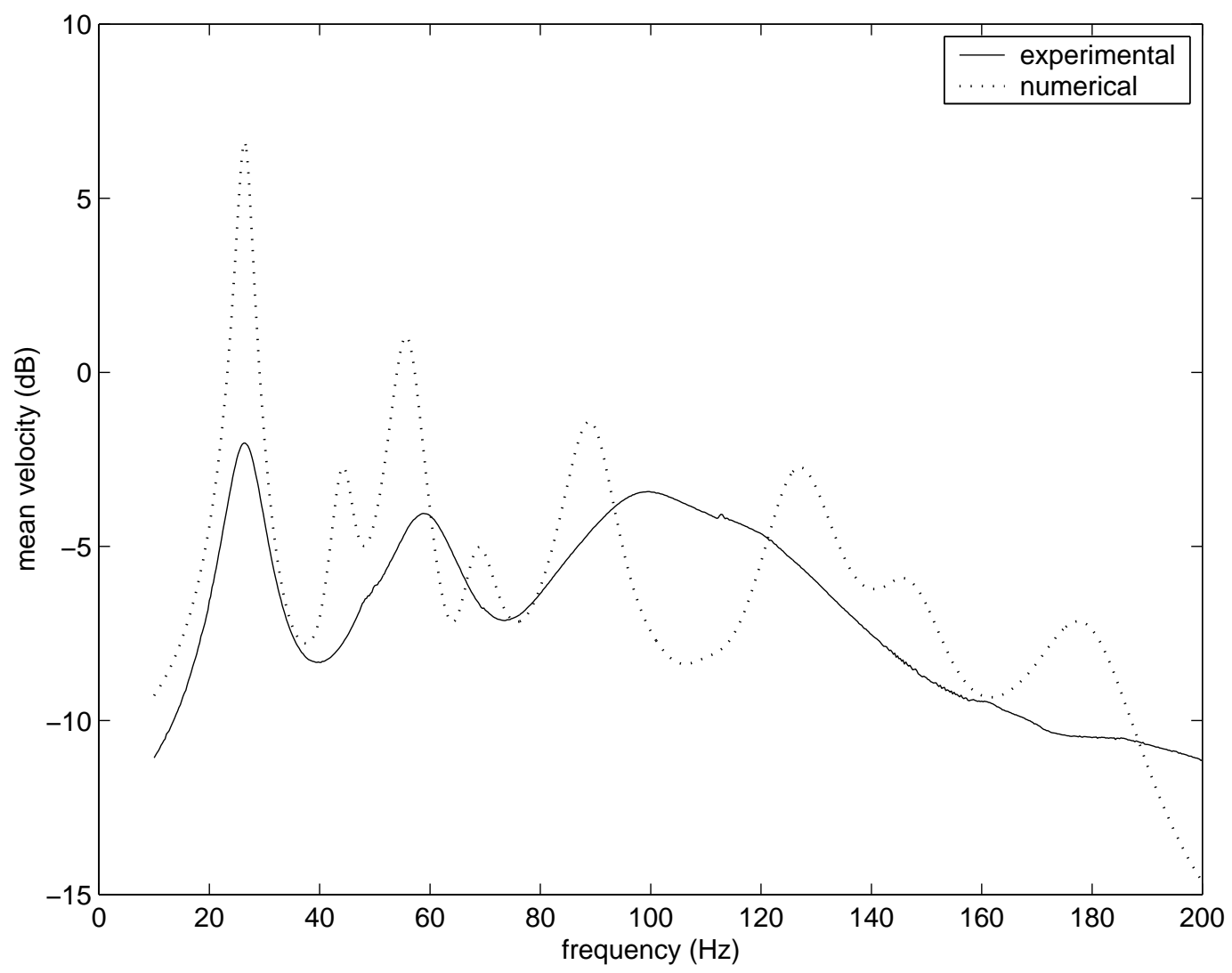

Figure 8: 